\title{
Hierarchical Self-Organization of Soft Patchy Nanoparticles into Morphologically Diverse Aggregates
}

\author{
Ioana C. Gârlea ${ }^{\mathrm{a}}$, Emanuela Bianchia ${ }^{\mathrm{a}, \mathrm{b}}$, Barbara Capone ${ }^{\mathrm{a}}$, Lorenzo Rovigatti ${ }^{\mathrm{a}, \mathrm{c}}$, Christos N. Likos ${ }^{\mathrm{a}}$ \\ ${ }^{a}$ Faculty of Physics, University of of Vienna, Boltzmanngasse 5, 1090 Vienna, Austria \\ ${ }^{b}$ Institut für Theoretische Physik, Technische Universität Wien, Wiedner Hauptstraße 8-10, 1040 Wien, Austria \\ ${ }^{c}$ Rudolf Peierls Centre for Theoretical Physics, University of Oxford, 1 Keble Road, Oxford, OX1 3NP, United Kingdom
}

\begin{abstract}
We present a concise review of the large variety of self-assembly scenarios observed in solutions of diblock copolymer stars with a solvophilic inner block and a solvophobic outer block. A variety of modeling approaches and simulation techniques at different levels of detail reveals that individual molecules assume configurations akin to patchy colloids, but with a patchiness that depends on physical parameters and can adjust to external stimuli such as temperature or $\mathrm{pH}$. These soft, patchy building blocks inter-associate at finite concentrations into micellar or gel-like solutions, including spherical and wormlike micelles, or they display macroscopic phase separation. The connections between single-molecule conformation and the structure of the concentrated solution are discussed, and coarse-grained strategies for these novel molecular entities are critically compared to one another.
\end{abstract}

Keywords: Star polymers, patchy colloids, diblock copolymers, spherical and wormlike micelles, gels

\section{Introduction}

Soft patchy nanoparticles are complex units at the nanoscale level that are characterized by soft and directional interactions. Examples of this class of particles can be mostly found in polymer-based systems: Janus-like polymer vesicles [1], patchy micelles emerging from the appropriate design of functionalised linear dendritic polymers [2], patchy nanoparticles with controllable symmetry resulting from the self-organization of block copolymer chains [3] or stars [4], block copolymer nanoparticles with microphase separation structures [5], hydrophobic dendrimers particles with dynamic hydrophobic patches [6] or colloids grafted with poor-solvent polymer brushes [7] are just some examples. Also DNA-coated colloids [8, 9] and emulsions [10], as well as DNA nanostars $[11,12]$ are usually considered within the same class of systems.

The present paper focuses on star polymers with functionalized arms, known as Telechelic Star Polymers (TSPs), and presents an overview of the hierarchical self-assembly properties of these complex units. Experimental realizations of such macromolecules are, e.g., end-functionalized, zwitterionic polybutadiene star polymers $[13,14]$. In particular, we consider TSPs whose arms are made of diblock copolymers - polymers made of two different blocks - composed of a solvophilic part, $A$, and a solvophobic part, $B$, where the latter is grafted onto a central core. The double nature of TSPs leads to a very rich assembly scenario when these macromolecules are exposed to selective solvents. Their ability to hierarchically self-assemble, first into soft patchy aggregates with different morphologies,

Email address: christos. likos@univie.ac.at (Christos N. Likos) and then into mesoscopic phases that are compatible with the functionalisation of the constituent units, makes TSPs excellent candidates as functionalised nanoparticles for smart materials.

At a single nanoparticle level, when exposed to a solvent, the intrinsic intramolecular interplay between entropic and enthalpic contributions leads to the formation of functionalised regions on the surface of the stars: when the density of solvophobic monomers is sufficiently high, either due to a high number of arms grafted on the anchoring point or to a high percentage of $A$ monomers per arm, the solvophobic regions tend to collapse on themselves forming patches, so that the contact with the solvent is minimized. While the solvophobic patches create a bonding pattern on the nanoparticle surface, the solvophilic core, due to the entropic repulsion between the B-monomers, acts as a soft substrate on which patches are distributed. The solvent quality, the temperature, the $\mathrm{pH}$, the number of arms per star and the ratio between solvophobic and solvophilic monomers per arm are the key quantities that completely allow to tune the single particle properties $[4,15]$. Once the physical and chemical parameters are set up, particles are either functionalised or not and the single particle properties are fully characterised.

At finite densities, when the nanoparticles interact with one another, the functionalised regions tend to coalesce creating an aggregation scenario where the single molecule properties are unaffected by the presence of other molecules [16]. In other words, the faster and tighter intra-star association as compared to the inter-star association leads to systems composed of equilibrium patchy aggregates, which can possibly interact with each other while keeping, on average, a well defined internal structure. The peculiar features of such self-assembled patchy aggregates are the extremely soft interactions and the possible 
fluctuations in the patch position/size; for this reason they are referred to as soft patchy nanoparticles.

Soft patchy units emerging in TSP systems combine directional interactions and low bonding valence with soft interactions and mobile patches. In contrast, hard patchy units are usually characterized by a pre-defined and fixed patchiness: for this class of systems, the number, the size and the geometrical arrangement of the patches cannot fluctuate. Additionally, the patches are usually such that the single bonding condition (one bond per patch) is guaranteed or, at least, favored. Hence, in hard patchy systems there exists a correspondence between the patch number and the bonding pattern; such a correspondence is an important feature determining the properties and the structure of the macroscopic phases. For our soft patchy nanoparticles the continuous fluctuations of the patch locations may lead to reversible modifications of the valence (patch number) and of the patch size; as a consequence the possibility of multiple bonding between the patches as well as the equilibrium patchiness at finite densities is controlled by the balance between the energy penalties associated to the distortion of the equilibrium patch arrangement and the energy gains due to the bonding between the patches.

In general, we can state that TSPs have a robust and flexible architecture and they possess the ability to self-assemble at different levels. At the single-molecule level, they first order as soft patchy colloids, which serve then as "soft Lego" for the emergence of larger structures. At the supramolecular level of self-assembly, the soft colloids assemble accordingly to their functionalities, spanning from gels, micellar structure up to complex crystal structures, such as for example diamond or cubic phases [4].

\section{Lattice models}

Considerable progress in understanding the properties of TSP $\mathrm{s}$ and their concentrated solutions has been achieved by simulations of lattice models; here, the pioneering work was that of Lo Verso et al. [17], who employed a lattice model for individual polymers, $f$ of which were anchored on a common center. Here, the model is built on a square lattice but sequential bonding between monomers is allowed not only along any of the 6 nearest neighbor directions but also along any of the 12 second neighbors and the 8 third neighbors, bringing the coordination of the lattice up to $z=26$ and thus emulating a continuum case. Lattice sites are either empty (modeling the solvent molecules) or occupied by monomers, which are of two types: $H$ for head or solvophilic and $T$ for tail or solvophobic. Accordingly, each arm of the star has the architecture $H_{m} T_{n}$, with $m$ and $n$ denoting the number of repulsive and attractive arms, respectively. The $f$ arms are all anchored on a common, $H$-type central bead, creating thereby star diblock copolymers coded as $H\left(H_{m} T_{n}\right)_{f}$, in obvious notation. All monomers are subject to excluded volume interactions, i.e., lattice site occupancy is at most unity. This is also the only condition for the $H-H$ and $H-T$ interactions, whereas $T-T$ interactions entail an attraction of depth $-\epsilon<0$ as long as they find themselves within the coordination shell $(z=26)$ of one another.
Extensive, grand canonical Monte-Carlo simulations of $H\left(H_{m} T_{n}\right)_{3}$-systems $(f=3)$ with $m+n=10$ revealed the following scenario [17]: for $n \geq m$, the system undergoes a macroscopic liquid-gas transition, terminating at a critical point with the critical temperature growing as the number of attractive endgroups increases. On the other hand, when $n<m$, the macroscopic phase separation disappears and the system forms micellar aggregates in which each TSP has collapsed its endpoints in one patch and several of such collapsed stars turn their patches into contact with one another, exposing their solvophilic parts to the solvent. This scenario has been fully confirmed by offlattice simulations showing the collapse of each star to a singlepatch configuration at sufficiently low temperatures $[4,18,19]$, as well as by recent simulations of micelle-like aggregates in the continuum, see Section 3.4. Upon further increase of the concentration, these spherical micelles begin to merge by fusing their attractive cores, forming thereby wormlike micelles, whose characteristics (length, stiffness, orientation) depend on the temperature as well as on the rigidity of the arms [17, 20]. The scenario has been confirmed also for the case of longer arms, $m+n=20$ [21]. Moving on to higher functionalities, $f=6$ and $f=10$, the self-assembly scenarios become richer. Indeed, although the systems still show macroscopic separation for $n \geq m$, for the opposite case, $n<m$, the emerging structure now depends also on the ratio $\alpha \equiv n /(n+m)$, i.e., the fraction of attractive monomers. Whereas for $\alpha=0.2$ again spherical micelles result, for $\alpha=0.4$ the system spontaneously forms a percolating, macroscopic gel [21]. These findings are in full agreement with subsequent and independent off-lattice simulations (see the following section), which demonstrate that for $\alpha=0.4$ the individual molecules adopt multipatch configurations, thus connecting into a macroscopic gel as concentration grows. On the other hand, for $\alpha=0.2$ all arms collapse onto a single patch, promoting therefore the stability of micelle-like organization as that shown in Section 3.4. Finally, we point out the possibility of emergence of ordered structures for TSPs with $n<m$, provided the arms are sufficiently rigid [22].

\section{Continuum models}

Proceeding from lattice to continuum models allows on the one hand to move to more realistic representations of homopolymers, and on the other hand to move to a coarse-grained representation of the system, that will allow, through a multiscale methodology, to more comprehensively explore the phase diagram of TSPs. Continuum models, which are not hindered by the discrete nature that affects the lattice ones and thus allow for a finer tuning of the parameters, are better fit to assess the resulting phase space. However, simulations of continuum models are also inherently slower than lattice simulations. Consequently, the results shown here pertain to either highly detailed small systems (one or few TSPs), or to bulk simulations of coarser-grained stars.

In order to assess the single particle properties as a function of the microscopic chemical and physical details, a full monomer representation of TSPs is used (Section 3.1), while to describe large scale systems, possibly at finite densities, a 
multiscale approach has been developed (Section 3.2); finally to further manage extended bulk systems at finite densities a second-level coarse-grained description has been put forward (Section 3.5). In the following, we describe the properties of isolated stars (Section 3.3), small assemblies of TSPs (Section 3.4) and TSPs at finite densities (Section 3.5), taking advantage of the appropriate system description.

\subsection{Full Monomer representation}

In a full-monomer representation, each TSP is made of $f$ diblock copolymer chains anchored to a central point through their solvophilic parts. Each chain is made of $N_{A}$ monomers of type $A$ (solvophilic) and $N_{B}$ monomers of type $B$ (solvophobic); note that the correspondence to the lattice model notation of the preceding Section is $H$ to $A$ and $T$ to $B$. The effect of the solvent is taken into account in an implicit way. Nearest (bonded) neighbours are connected through a standard FENE potential. All the repulsive interactions acting between both bonded and non-bonded $A-A$ and $A-B$ pairs are modelled through a generalised Lennard-Jones (LJ) potential, truncated and shifted in the Weeks-Chandler-Andersen (WCA) fashion:

$$
V_{\mathrm{WCA}}(r)= \begin{cases}4 \epsilon\left[\left(\frac{\sigma}{r}\right)^{48}-\left(\frac{\sigma}{r}\right)^{24}\right]+\epsilon & \text { if } r<r_{c}, \\ 0 & \text { otherwise, }\end{cases}
$$

where $\sigma$ is the monomer diameter, $\epsilon$ is the depth of the potential well before shifting and $r_{c}=2^{1 / 24} \sigma \approx 1.03 \sigma$ is the cutoff radius.

The effective attraction between the terminal solvophobic monomers is provided by the attractive tail of the same generalised LJ potential as in Eq. (1), rescaled by the dimensionless number $\lambda$,

$$
V_{\mathrm{attr}}(r)= \begin{cases}V_{\mathrm{WCA}}(r)-\epsilon \lambda & \text { if } r<r_{c}, \\ 4 \lambda \epsilon\left[\left(\frac{\sigma}{r}\right)^{48}-\left(\frac{\sigma}{r}\right)^{24}\right] & \text { otherwise. }\end{cases}
$$

The role of $\lambda$ is to control the strength of the attraction between $B-B$ pairs, effectively acting as the inverse temperature; evidently, for $\lambda=1$ we recover the full, generalised Lennard-Jones potential. The large values of the exponents in the generalised Lennard-Jones interaction have been chosen to make the interaction more short-ranged and hence computationally faster. Additional details about the model can be found in Ref. [15].

In what follows, lengths are measured in units of $\sigma$, energies in units of $\epsilon$ and masses in units of the monomer mass, $m$. The main physical parameter of the model is the ratio between the number of solvophobic monomers and the total number of monomers, $\alpha=N_{B} /\left(N_{A}+N_{B}\right)$. The temperature is kept fixed at a value $k_{B} T=0.5$, with the strength of the attraction, which embodies the main chemical parameter, being controlled solely by $\lambda$.

\subsection{Multiblob Multiscale Methodology}

To extend the analysis from the single molecule behavior to the full phase diagram, the development of both a theoretical model and of suitable simulation techniques appears to be crucial. The billions of monomeric units involved in the simulations of polymers in the semi-dilute regime require the usage of a multiscale coarse-grained representation that needs to be precise and back-traceable onto the microscopic system, thus allowing to bridge the gap between theoretical predictions and experimental realizations of the system. This implies the use of a methodology that bridges over length scales that allows us, starting from few chemical and physical details, to predict global properties of the system and to test the results upon an experimental verification. The coarse-graining methodology starts from a microscopic representation of polymers at infinite dilution [4]. For every $\mathrm{pH}$, solvent quality or temperature, a set of effective interactions is derived from the simulation of two linear chains split into dimers [23]: infinite dilution simulations allow to derive the set of intramolecular and intermolecular pair distribution functions between the four constituents of the dimers that, by means of an analytical inversion, give a (temperature dependent) set of effective potentials that represent a group of monomers. Such a methodology has been extensively tested in the last few years, and it was proven to reproduce microscopical predictions, both when tested on full monomer simulations in complicated geometrical constraints (e.g., homopolymer brushes [24]) and when compared to experimental results for diblock copolymers in the semi-dilute regime [25]. The effective interactions between blobs are determined by a first principles inversion procedure, which was obtained for diblock copolymers [25, 26], generalizing the method used earlier for the simple dumbbell representation of diblock copolymers [27, 28, 29].

In a microscopic representation, given a TSP, each $A B$ diblock arm with a total number of monomers $M$ contains $M_{A}$ monomers of kind A and $M_{B}$ monomers of kind $B$. As mentioned, in the multiblob representation, each of the two blocks, $A$ and $B$, is mapped onto $n_{A}$ and $n_{B}$ blobs respectively, where every blob contains a number $m_{A}=M_{A} / n_{A}$ or $m_{B}=M_{B} / n_{B}$ of monomers of the underlying microscopic model. Each blob has a radius of gyration $r_{g \gamma}=a b_{\gamma} m_{\gamma}^{\nu_{\gamma}}$ with the subscript $\gamma=A, B$ denoting the monomer species, $v_{\gamma}$ being the scaling exponent of the $\gamma$ species, $a$ the monomer size and $b_{\gamma}$ being model-specific, numerical coefficients of order unity. Since the asymmetry ratio $\alpha$ can be rewritten in terms of blobs and their radii of gyration, we obtain a relation between the asymmetry ratio, the number of $A$ and $B$ blobs, and the radii of gyration $r_{g A}, r_{g B}$.

The three nonbonded potentials, $V_{\alpha \beta}(r)$, act between all blobs whereas the tethering potentials, $\phi_{\alpha \beta}(r)$, only between sequential blobs along a star arm; here, the centers of two blobs of types $\alpha$ and $\beta,(\alpha, \beta=A, B)$, separated by a distance $r$, are the effective coordinates. These potentials are made of a superposition of repulsive and attractive terms of the form:

$$
V_{\alpha \beta}(r)=\sum_{j} A_{\alpha \beta}^{(j)} \exp \left[-B_{\alpha \beta}^{(j)}\left(r / r_{g}\right)^{2}\right],
$$

for the nonbonded interactions and of the form

$$
\varphi_{\alpha \beta}(r)=\sum_{j} C_{\alpha \beta}^{(j)}\left(r / r_{g}-D_{\alpha \beta}^{(j)}\right)^{2}
$$

for the tethering potentials, where the number of terms in the sum over $j$ depends on the combination $\alpha \beta$. Moreover, the val- 


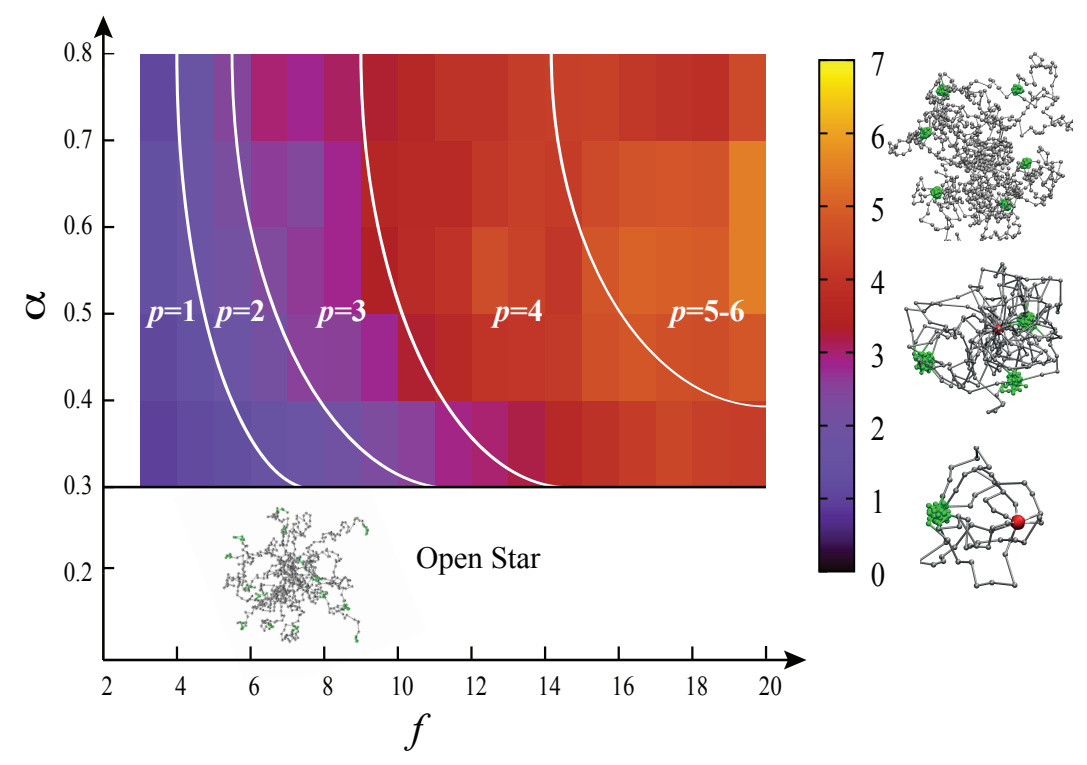

Figure 1: Single-molecule conformational state diagram of telechelic star polymers as a function of the number of chains per molecule $f$ and asymmetry ratio $\alpha$. The quantity $p$ stands for the number of multiarm patches. (Redrawn from Ref. [4].)

ues of the parameters $A_{\alpha \beta}^{(j)}, B_{\alpha \beta}^{(j)}, C_{\alpha \beta}^{(j)}$ and $D_{\alpha \beta}^{(j)}$ depend on the $\alpha \beta$ combination as well. For further details on their values and also on the derivation of these potentials, we refer the reader to the original publications [23, 25, 26, 27, 28, 29].

The methodology described in this Section is based on the assumption that the molecular weights of the polymer segments, underlying each blob, are high enough to guarantee that the polymer is in the scaling limit. This renders the methodology independent from the underlying model and from the particular microscopic length of the polymer used, thus allowing to access information for a class of polymers in a given point of the phase space.

\subsection{Isolated single stars: intra-molecular self-organization}

The first step towards an understanding of the bulk behaviour of TSPs is the characterisation of the structure of a single, isolated star. We have recently shown how the tunability of such a system can be widely extended by controlling the physical parameters of the star (that is, $f, N_{A}, N_{B}$ and $\alpha$ ), as well as the chemical parameters of the solution [15], building upon earlier results that hinted to the varieties of intramolecular association patterns of TSPs [18, 19]. In fact, the dependence of the star structure on the latter is of great importance for applications, since our results show that changes in temperature and/or solvent quality make it possible to reliably change the number and size of the attractive patches. This, in turn, allows to steer the mesoscopic self-assembly behaviour without modifying the microscopic constituent.

For a fixed state point, the single-star properties are completely determined by the molecular composition, i.e. by the number of arms $f$, the number of monomers $N_{A}$ and $N_{B}$ and the percentage $\alpha$ of attractive monomers per arm. In addition, in the scaling regime the dependence on the number of monomers drops, and $f$ and $\alpha$ are the only parameters influencing the competition between entropic and enthalpic terms [4, 16]. Hence, fixed-thermodynamic-point state diagrams can be represented as planes in the $(f, \alpha)$ space [4]. Fig. 1 shows such a state diagram, obtained through simulations of coarse-grained single stars [4].

By choosing, in a selective solvent, the right combination of functionality $f$ and percentage of attractive monomers $\alpha$, a single TSP self-assembles into a soft patchy particle that preserves its character (number of patches, average angle between the patches, average extension of the patches from the anchor point) upon increasing density, from the extremely dilute case up to the semi-dilute regime (see Section 3.5). The aggregation into these soft patchy structures is fully determined by the balance between the entropic contributions, arising from the intra-star repulsions in the core of the stars, and the competing enthalpic terms, arising from the solvophobic nature of the terminal monomeric- or blob-units.

As put already forward in Ref. [4], in the case in which the thermal, solvophobic tails lie slightly below their $\Theta$-point, one finds that, for $\alpha<0.3$, the macromolecules remain in an open star configuration even when the number of arms is increased. By contrast, for $\alpha \geq 0.3$ the macromolecules always self-assemble into soft patchy particles. Moreover, for a fixed functionality $f$, the number of patches $p$ can be tuned by changing $\alpha$. Alternatively, if $\alpha$ is kept constant the number of patches can be selected by varying the functionality of the star.

A different path to steer the self-organisation of TSPs for a given $(f, \alpha)$ combination is to change the solvent quality, i.e., to influence the attraction between the monomers of the endblocks. Indeed, solvent quality can be seen as a third external parameter that can be used not only to control the formation of particles with a given number of patches, but also to tune the radial and angular flexibility of those functionalized domains. 


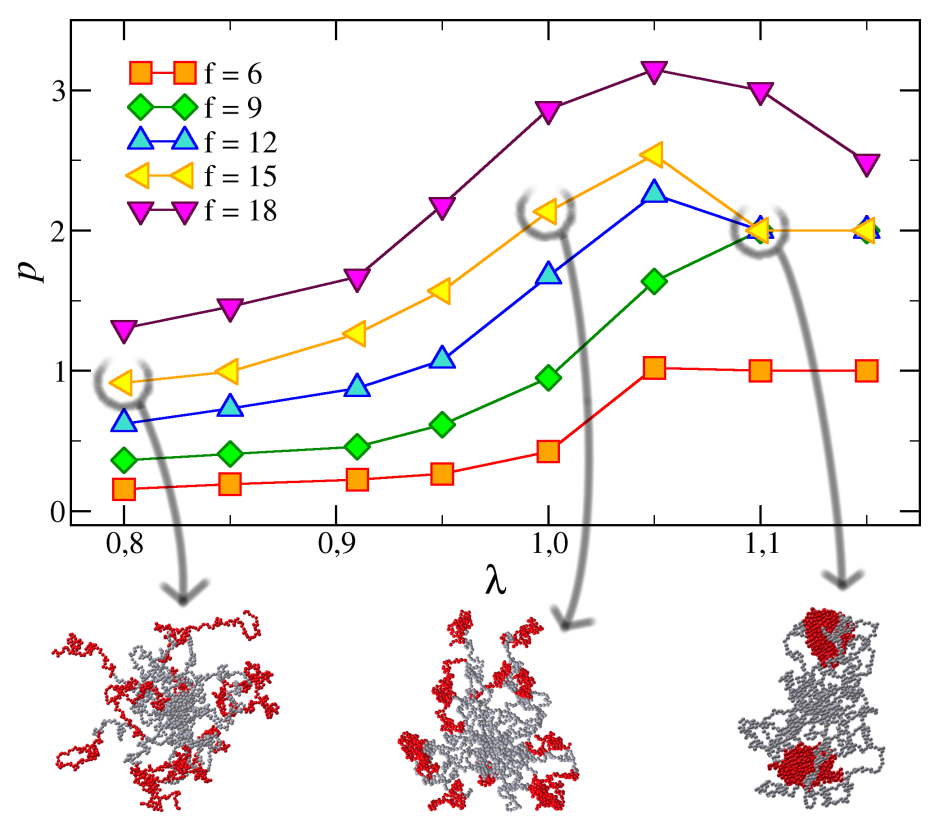

Figure 2: Number of patches as a function of $\lambda$ for fixed $\alpha=0.5$ and several values of $f$. The snapshots show three representative configurations for $f=15$ at three different values of $\lambda$.

Fig. 2 shows the effect of temperature (here embodied by $\lambda^{-1}$ ) on the number of patches for $\alpha=0.5$ and for different functionalities $f$. The qualitative process is sketched at the bottom of the figure with simulation snapshots: as $\lambda(T)$ increases (decreases), the arms start to collapse on themselves, forming patch-like aggregates. At intermediate $\lambda$-values these aggregates continuously form and break apart. By contrast, for the highest values of $\lambda$ the patches are completely formed and stable [15]. The detailed full-monomer description summarized here can be also used as a starting point for a further bottomup coarse-graining, giving rise to quantitative coarse-grained models with the level of description as the one presented in Section 3.2.

\subsection{Formation of micellar aggregates}

As discussed in Section 2, lattice simulations have shown that, for specific combinations of physical and chemical parameters, TSPs form stable micelles with a relatively broad size distribution [21]. Here we perform off-lattice simulations of micellar aggregates made of $N_{s}$ TSPs to investigate their mechanical stability, as well as the dependence of their size and shape on $N_{s}$.

We study TSPs with $\alpha=0.2, N_{A}=24$ (and hence $N_{B}=6$ ), $\lambda=2$ and $f \in\{3,4,5\}$. In Fig. 3(a)-(c), we show representative simulation snapshots for all the investigated stars. For the chosen combination of parameters we observe the formation of either one or two patches, depending on the functionality $f$. TSPs with $f=3$ and $f=4$ have always a single patch, which is invariably composed of all the available solvophobic monomers. By contrast, if we move to higher number of arms, we see that the size of a patch cannot grow indefinitely. Indeed, after a certain size, the steric hindrance of the solvophilic monomers of the arms that compose the patch is so high that it prevents any other arm from joining the aggregate. As a consequence, we see that the 5-arm TSP exhibits two possible self-aggregation configurations [19]: either the final structure has two attractive patches (one in which three arms are bond together and one containing the remaining two, see Fig. 3(c), or one structure where four arms form together an attractive patch and the fifth one is free. Incidentally, we note that the latter configuration is less likely.

Lattice simulations have shown that the basic building block of micelles made of TSPs is a "watermelon" aggregate, which is a TSP having all the solvophobic beads forming one single attractive patch lying opposite to the anchoring point [20]. Therefore, we exclude the $f=5$ star and resort to building micellelike aggregates out of $f=3$ and $f=4$ TSPs only. In order to do so, we put $N_{s}$ equilibrated watermelons in the simulation box and add a spring-like potential acting between the centers of mass of each pair of attractive patches. We run the simulation till all the attractive patches are in contact with each other and then switch off the biasing potential. Finally, we let the formed aggregate equilibrate and then start gathering statistics.

We investigated aggregates formed out of a minimum of 2 and a maximum of 30 stars. Fig. 3(d)-(k) shows simulation snapshots for aggregates of different size, formed by both $f=3$ and $f=4$ TSPs. Within an aggregate all solvophobic beads are clustered together in its center, whereas the anchors lay on the outer side of the structure. All the assembled aggregates were found to be stable over time, with their shape being also preserved.

\subsection{TSPs at finite densities}

The development of a reliable multiscale strategy allowed to perform extensive studies starting from a very dilute up to 
(a)

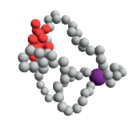

(d)

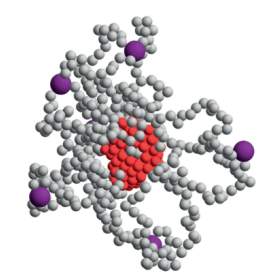

(h)

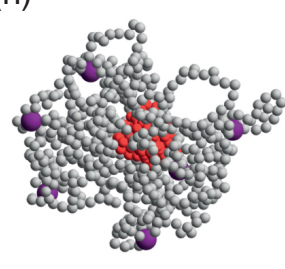

(b)

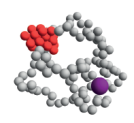

(e)

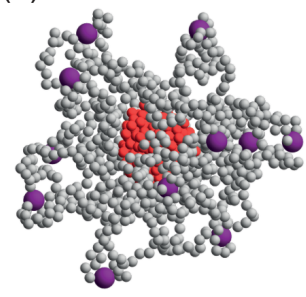

(i)

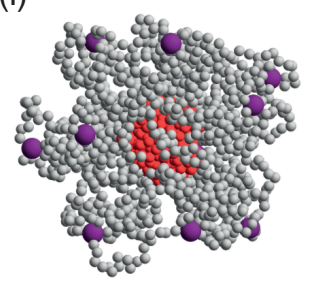

(c)

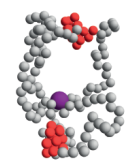

(f)
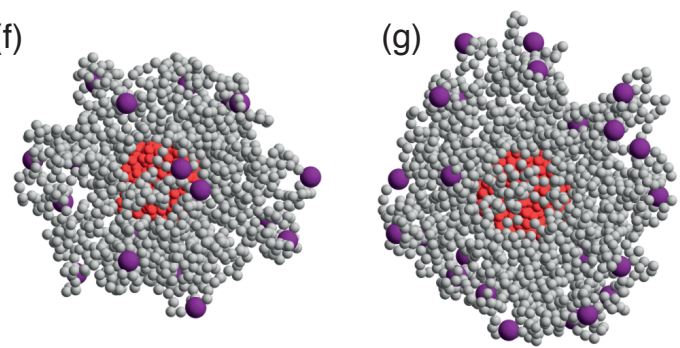

(j)
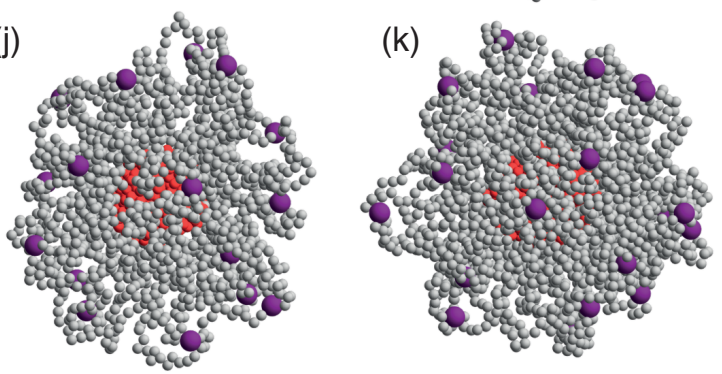

Figure 3: Snapshots of single TSP aggregates and micellar aggregates. The top row shows single TSP aggregates obtained for stars containing (a) 3 arms, (b) 4 arms, and (c) 5 arms. The middle and bottom rows show snapshots of micelles containing 6 TSPs [(d) and (h)], 12 TSPs [(e) and (i)], 18 TSPs [(f) and (j)], and 24 TSPs $[(\mathrm{g})$ and $(\mathrm{k})]$. In the middle row the TSPs composing the aggregates have $f=3$ arms, whereas in the bottom row $f=4$.

the semi-dilute regime, thus assessing the sturdiness of the single particle self-aggregation behaviour upon perturbations such as the increase in density and the consequent interactions with other macromolecules. The single-star self-aggregating scenario was shown to be very robust: the behavior of TSPs at finite densities shows a well-defined patchiness per unit as a function of the functionality $f$ and the asymmetry ratio $\alpha$ [16]. In particular, it was shown that the average number of patches per star, the average distance between a patch and its core as well as the average angular distribution of the patches around their center are preserved upon augmenting the density up to relatively high densities. While the number of patches is preserved on changing $f$, a change in $\alpha$ affects the angular flexibility and the softness of the elongation of the patches, as well as the size of the patch [16]. Within the multiblob description, TSPs stars in solution at various densities showed to retain the zero density patchiness for a wide range of $(f, \alpha)$ combinations [16].

As TSPs are able to self-assemble into robust soft patchy units, they are prone to undergo a subsequent self-organization process where the final phase is compatible with the functionalization of the self-assembled units. Starting from the extremely dilute phase and increasing the density of the stars in solution, TSPs are prone to form aggregates that might cluster into percolating, gel-like networks, where the latter are defined as all those systems in which stars assemble in amorphous percolating structures and where the motion of the anchoring point of the stars is extremely slow (see Fig. 4, upper panel). The resulting gel phase is formed via the fusion of the patches of the single stars into larger patches shared by two or more stars. We note that the route towards gel formation in these systems has not been systematically studied. In contrast to lattice simulations, where no sign of phase transitions has been observed [21], in the continuum the possible presence of a gasliquid transition definitely requires more investigation.

The possibility of tuning the number and the equilibrium arrangement of the patches in combination with the capability of these patchy assemblies to maintain their internal structure at finite density, strongly motivated the investigation of bulk systems at a second coarse-grained level. A simple patchy model that keeps close connection to the polymer-based complex units, while dealing with pair interaction potentials between few effective entities has been recently introduced [30]. The movements of the patches are controlled by two flexibility parameters: one describes the patch elongation along the radial direction, while the other one is associated to the angular patch freedom. In this way, both the patch-core distance and the patch-patch angle within the same particle are allowed to fluctuate around their respective equilibrium values. The internal energy of each effective particle is the result of pair interactions occurring between the single entities, either cores or patches, belonging either to the same particle or to two different particles. A deep insight in the self-organisation pathways of these complex units can be obtained via a systematic variation of the two flexibility parameters. The described model has allowed to explore the role of softness and patch rearrangement on the formation of gel networks at a coarse-grained level where both features can be controlled by suitably chosen parameters. The 

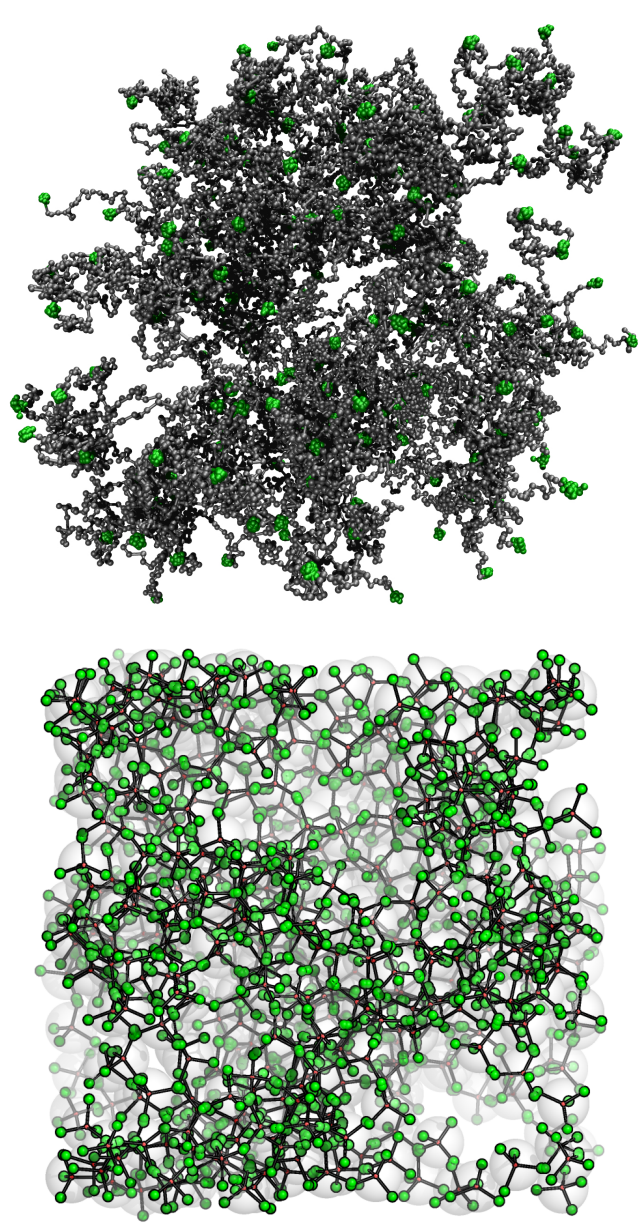

Figure 4: Snapshot of a percolating gels formed by TSPs: on the upper panel, telechelic star polymers within the multiblob description with $f=5$ arms and $\alpha=0.6$ are shown; on the bottom panel, soft patchy units within the second level coarse-grained description. The green spheres represent attractive endmonomers (upper panel) or attractive patches (bottom panel).

self-organisation scenarios of this model were shown to be dictated by the competition between the energy penalties associated to the patch fluctuations and the energy gains due to the patch bonding. Among the emerging gels, significantly different morphologies could be identified: for relatively rigid units, an homogeneous structure was observed, while on increasing the patch flexibility, voids emerged in the gel structure due to clustering of several patches; interestingly, on further increasing the patch flexibility, the morphology of the network became again homogeneous (see Fig. 4, bottom panel).

Finally, patchiness is preserved upon very high density rendering TSPs a perfect tunable soft building block to assemble ordered structures compatible with the self-assembled functionalisation of the soft colloidal macromolecules. Such a property has been exploited to demonstrate the mechanical stability of different crystals (simple cubic and diamond lattices) in Ref. [4], compatible with the single particle functionalisation.

\section{Conclusions}

Telechelic star polymers self-organize into a large variety of soft patchy nanoparticles, whose conformations and patchiness can be tuned and modified either by choosing the functionality and degree of amphiphilicity or even in situ, by thermally affecting the solubility of the attractive end-blocks. The conformation of the single molecule largely affects the emerging mesoscopic structure of the concentrated solution: whereas single-patch TSPs tend to form spherical micelles that further organize into wormlike micelles as the concentration grows, multipatch particles form percolating gels, and tetrahedrally coordinated TSPs can support the (mechanical) stability of fourfold-coordinated crystals such as the diamond lattice. The ramifications of these various forms of self-organization for the rheological response of such solutions [31, 32] (viscosity, nonNewtonian behavior, shear-banding etc.) is the subject of current investigations.

We note that, while in other systems with mobile and flexible bonds the patches can form one and only one bond, irrespective of how flexible and soft the particle bonds are [12, 33, 34], the described coarse-grained model for polymer-based patchy units is designed to consider the possibility of multiple bonding between the patches as well as possible energy penalties associated to the distortion of the equilibrium patch arrangement [30]. It is such a competition that governs the emergence of different disordered phases or, possibly, crystalline ones.

Whereas the equilibrium properties of concentrated systems of TSPs are, due to the interest in the variety of assembly scenarios they present, the subject of ongoing investigations, current research is also increasingly turning its attention to the behavior of these systems under non-equilibrium conditions. Future challenges include the investigation of the behavior of the patchiness of individuals TSPs under (shear) flow and its influence on their rotation- and tumbling-dynamics in comparison with their non-fucntionalized counterparts, as well as the mechanical and rheological properties of concentrated solutions of the same. In particular, the stress distribution, dynamical rearrangements and the thixotropic behavior of the variety of TSP-gels is a topic deserving further investigations and offering promises for the designed control of the viscoelastic properties of the material.

\section{Acknowledgements}

E.B. acknowledges financial support from the Austrian Science Fund (FWF) through an Elise Richter Fellowship, Grant No. V249-N27. B.C. acknowledges financial support through an APART-Fellowship of the Austrian Academy of Sciences (ÖAW), Grant No. 11723. L.R. acknowledges financial support from the Austrian Science Fund (FWF) through a LiseMeitner Fellowship, Grant No. M 1650-N27 and from the European Commission through the Marie Skłodowska-Curie Fellowship No. 702298-DELTAS. This work has been supported by the Marie Skłodowska-Curie European Training Network COLLDENSE (H2020-MCSA-ITN-2014, Grant No. 642774). 
Computer time at the Vienna Scientific Cluster (VSC) is also gratefully acknowledged.

[1] F. Wurm, A. F. M. Kilbinger, Polymeric Janus particles, Angewandte Chemie International Edition 48 (2009) 8412.

[2] Z. Poon, S. Chen, A. C. Engler, H. Lee, E. Atas, G. von Maltzahn, S. N Bhatia, P. T. Hammond, Ligand-clustered patchy nanoparticles for modulated cellular uptake and in vivo tumor targetin, Angewandte Chemie International Edition 49 (2010) 7266.

[3] J. Zhang, Z.-Y. Lu, Z.-Y. Sun, A possible route to fabricate patchy nanoparticles via self-assembly of a multiblock copolymer chain in one step, Soft Matter 7 (2011) 9944-9950.

[4] ${ }^{* *}$ B. Capone, I. Coluzza, F. Lo Verso, C. N. Likos, R. Blaak, Telechelic star polymers as self-assembling units from the molecular to the macroscopic scale, Phys. Rev. Lett. 109 (2012) 238301.

[5] ${ }^{*}$ T. Higuchi, A. Tajima, K. Motoyoshi, H. Yabu, M. Shimomura, Frustrated phases of block copolymers in nanoparticles, Angewandte Chemie International Edition 47 (2008) 8044.

[6] ${ }^{* *}$ M. T. Hermans, M. A. C. Broeren, N. Gomopoulos, P. van der Schoot, M. H. P. van Genderen, N. A. J. M. Sommerdijk, G. Fytas, E. W. Meijer, Self-assembly of soft nanoparticles with tunable patchiness, Nature Nanotechnology 4 (2009) 721.

[7] ${ }^{* *}$ R. M. Chuoeiri, E. Galati, H. Thérien-Aubin, A. Klinkova, E. M. Larin, A. Quejereta-Fernández, L. Han, H. L. Xin, O. Gang, E. B. Zhulina, M. Rubinstein, E. Kumacheva, Surface patterning of nanoparticles with polymer patches, Nature 538 (2016) 79.

[8] *B. M. Mladek, J. Fornleitner, F. J. Martinez-Verachoechea, A. Dawid, D. Frenkel, Quantitative prediction of the phase diagram of DNAfunctionalized nanosized colloids, Phys. Rev. Lett. 108 (2012) 268301.

[9] ${ }^{*}$ S. Angioletti-Uberti, P. Varilly, B. M. Mognetti, D. Frenkel, Mobile linkers on DNA-coated colloids: Valency without patches, Phys. Rev. Lett. 113 (2013) 128303.

[10] L. Feng, L.-L. Pontani, R. Dreyfus, P. Chaikin, J. Brujic, Specificity, flexibility and valence of DNA bonds guide emulsion architecture, Soft Matter 9 (2013) 9816.

[11] L. Rovigatti, F. Smallenburg, F. Romano, F. Sciortino, Gels of DNA nanostars never crystallize, ACS Nano 8 (2014) 3567.

[12] E. Locatelli, P. H. Handle, C. N. Likos, F. Sciortino, L. Rovigatti, Condensation and demixing in solutions of DNA nanostars and their mixtures, ACS Nano 11 (2017) 2094.

[13] ${ }^{* *}$ M. Pitsikalis, N. Hadjichristidis, J. W. Mays, Model mono-, di- and tri- $\omega$-functionalized three-arm star polybutadienes. association behavior in dilute solution by dynamic light scattering and viscometry, Macromolecules 29 (1996) 179.

[14] *D. Vlassopoulos, T. Pakula, G. Fytas, M. Pitsikalis, N. Hadjichristidis, Controlling the self-assembly and dynamic response of star polymers by selective telechelic functionalization, J. Chem. Phys. 111 (1999) 1760.

[15] * L. Rovigatti, B. Capone, C. N. Likos, Soft self-assembled nanoparticles with temperature-dependent properties, Nanoscale 8 (2016) 3288-3295.

[16] * B. Capone, I. Coluzza, R. Blaak, F. Lo Verso, C. N. Likos, Hierarchical self-assembly of telechelic star polymers: from soft patchy particles to gels and diamond crystals, New Journal of Physics 15 (2013) 095002.

[17] *F. Lo Verso, A. Z. Panagiotopoulos, C. N. Likos, Aggregation phenomena in telechelic star polymer solutions, Phys. Rev. E 79 (2009) 010401(R).

[18] F. Lo Verso, C. N. Likos, C. Mayer, H. Löwen, Collapse of telechelic star polymers into watermelon structures, Phys. Rev. Lett. 96 (2006) 187802.

[19] F. Lo Verso, C. N. Likos, H. Löwen, Computer simulation of thermally sensitive telechelic star polymers, J. Phys. Chem. C 111 (2007) 15803.

[20] C. Koch, A. Z. Panagiotopoulos, F. Lo Verso, C. N. Likos, Customizing wormlike mesoscale structures via self-assembly of amphiphilic star polymers, Soft Matter 11 (2015) 3530-3535.

[21] C. Koch, C. N. Likos, A. Z. Panagiotopoulos, F. Lo Verso, Self-assembly scenarios of block copolymer stars, Mol. Phys. 109 (2011) 3049-3060.

[22] C. Koch, A. Z. Panagiotopoulos, F. Lo Verso, C. N. Likos, Phase behavior of rigid, amphiphilic star polymers, Soft Matter 9 (2013) 7424.

[23] ** B. M. Ladanyi, D. Chandler, New type of cluster theory for molecular fluids - interaction site cluster expansion, J. Chem. Phys. 62 (1975) 4308.

[24] I. Coluzza, B. Capone, J.-P. Hansen, Rescaling of structural length scales for "soft effective segment" representations of polymers in good solvent, Soft Matter 7 (2011) 5255.

[25] B. Capone, J.-P. Hansen, I. Coluzza, Competing micellar and cylindrical phases in semi-dilute diblock copolymer solutions, Soft Matter 6 (2010) 6075 .

[26] B. Capone, I. Coluzza, J.-P. Hansen, A systematic coarse-graining strategy for semi-dilute copolymer solutions: from monomers to micelles, J. Phys.: Condens. Matter 23 (2011) 194102.

[27] C. I. Addison, J.-P. Hansen, V. Krakoviack, A. A. Louis, Coarse-graining diblock copolymer solutions: a macromolecular version of the widomrowlinson model, Mol. Phys. 103 (2005) 3045.

[28] ${ }^{* *}$ C. Pierleoni, C. Addison, J.-P. Hansen, V. Krakoviack, Multiscale coarse graining of diblock copolymer self-assembly: From monomers to ordered micelles, Phys. Rev. Lett. 96 (2006) 128302.

[29] B. Capone, C. Pierleoni, J.-P. Hansen, V. Krakoviack, Entropic selfassembly of diblock copolymers into disordered and ordered micellar phases, J. Phys. Chem. B 113 (2009) 3629.

[30] *E. Bianchi, B. Capone, G. Kahl, C. N. Likos, Soft-patchy nanoparticles: modeling and self-organization, Faraday Discuss. 181 (2015) 123-138.

[31] ${ }^{* *}$ F. Clément, A. Johner, J.-F. Johanny, A. N. Semenov, Stress relaxation in telechelic gels. 1. sticker extraction, Macromolecules 33 (2000) 6148.

[32] *F. J. Stadler, W. Pyckhout-Hintzen, J.-M. Schumers, C.-A. Fustin, J.F. Gohy, C. Bailly, Linear viscoelastic rheology of moderately entangled telechelic polybutadiene temporary networks, Macromolecules 42 (2009) 6181.

[33] *F. Smallenburg, F. Sciortino, Liquids more stable than crystals in particles with limited valence and flexible bonds, Nature Physics 9 (2013) 554.

[34] ${ }^{* *}$ F. Smallenburg, L. Filion, F. Sciortino, Erasing no-man's land by thermodynamically stabilizing the liquid-liquid transition in tetrahedral particles, Nature Physics 10 (2014) 653. 